\title{
Simulation based Analysis of Adhoc Routing Protocol in Urban and Highway Scenario of VANET
}

\author{
Pranav Kumar Singh \\ Assistant Professor \\ Department of Computer Science \& \\ Engineering \\ Central Institute of Technology, \\ Kokrajhar, Assam-783370 (India)
}

\author{
Kapang Lego \\ Assistant Professor \\ Department of Computer Science \& \\ Engineering \\ NERIST, Nirjuli, \\ Arunachal Pradesh (India)
}

\author{
Dr. Themrichon Tuithung \\ Professor \\ Department of Computer Science \& \\ Engineering \\ NERIST, Nirjuli, \\ Arunachal Pradesh (India)
}

\begin{abstract}
VANET (Vehicular Adhoc Network) research field is growing very fast. It has to serves a wide range of applications under different scenario (City, Highway). It has various challenges to adopt the protocols that can serve in different topology and scenario. This paper presents a comparative study of the adhoc routing protocol in urban and highway scenario of VANET environments. The main objective of Vehicular Ad-Hoc Networks is to build a robust network between mobile vehicles so that vehicles can talk to each other for the safety of human beings. VANET hits the protocol's strength due to its highly dynamic features, thus in testing a protocol suitable for VANET implementation we have selected two different scenarios of traffic, and three adhoc routing protocols AODV, OLSR and DSR. Packet Delivery Ratio and End To End delay are the metrics used for performance analysis of the adhoc routing protocols.
\end{abstract}

\section{Keywords}

AODV, OLSR, DSR, PDR, E2E Delay, MOVE.

\section{INTRODUCTION}

VANET is a special case of the general MANET to provide communications among nearby vehicles and between vehicles and nearby fixed roadside equipments. VANET networks, nodes are characterized by high dynamic and mobility, in addition to the high rate of topology changes and density variability [5]. VANETs are a subset of MANETs (Mobile Ad-hoc NETworks) in which communication nodes are mainly vehicles. As such, this kind of network should deal with a great number of highly mobile nodes, eventually dispersed in different roads. In VANETs, vehicles can communicate each other (V2V, Vehicle-to-Vehicle communications). They can connect to an infrastructure (V2I, Vehicle-to-Infrastructure) or Infrastructure to Vehicle (I2V) to get some service. This infrastructure is assumed to be located along the roads.

Some motivations of the promising VANET technology include, Increase traveler safety, Enhance traveler mobility, Decrease travelling time, Conserve energy and protect the environment, Magnify transportation system efficiency, Boost on-board luxury but it is not enough many other services can be served by using this technology.
The creation of Vehicular Ad Hoc Networks (VANET) has spawn much interest all over the world, in German there is the FleetNet[1] project and in Japan the ITS(Intelligent Transportation System) project. Vehicular ad hoc networks are also known under a number of different terms such as Inter Vehicle communication (IVC), Dedicated Short Range Communication (DSRC) or Wireless Access in Vehicular Environments (WAVE) [2]. The goal of most of these projects is to create new network algorithms or modify the existing for use in a vehicular environment. In the future vehicular ad hoc networks will assist the drivers of vehicles and help to create safer roads by reducing the number of automobile accidents.

Vehicles equipped with wireless communication technologies and acting like computer nodes will be on the road soon and this will revolutionize the concept of travelling. VANETs bring lots of possibilities for new range of applications which will not only make the travel safer but fun as well.

As VANET is a subset of MANET. The technology and protocols for MANETs need to be evaluated carefully and then adapted in order to be used in VANET context. MANET and VANET, both are mobile networks but they mainly differs in terms of their mobility pattern of VANET nodes is such that they move on specific paths (roads) and hence not in random direction. In VANET the nodes are car having sufficient storage capacity and high processing power unlike MANET nodes lack in storage and processing power.

Although there are many proposed solutions for routing in VANET, it is still unclear as to what specific characteristics VANET routing protocols should possess, since none of the proposed solutions has given the clear idea about the performance of routing protocol in both urban and highway scenario. To shed light on these issues, we analyze some of the most important metrics in VANET. Node density, Movement Patterns, and node velocity are the main challenge to develop a suitable protocol for VANET.

In this paper, as we told that VANET is a subset of MANET we are trying to analyze the performance of three highly adopted adhoc routing protocols AODV, OLSR and DSR in two different scenario of traffic i.e. Urban and Highway to check that whether they are applicable in VANET or not.

The performance of the proposed protocol has been studied using simulation tools mainly Network Simulator (NS) and MOVE (MObility model generator for VEhicular networks) over SUMO. 
The figure given below is a example of VANET scenario.

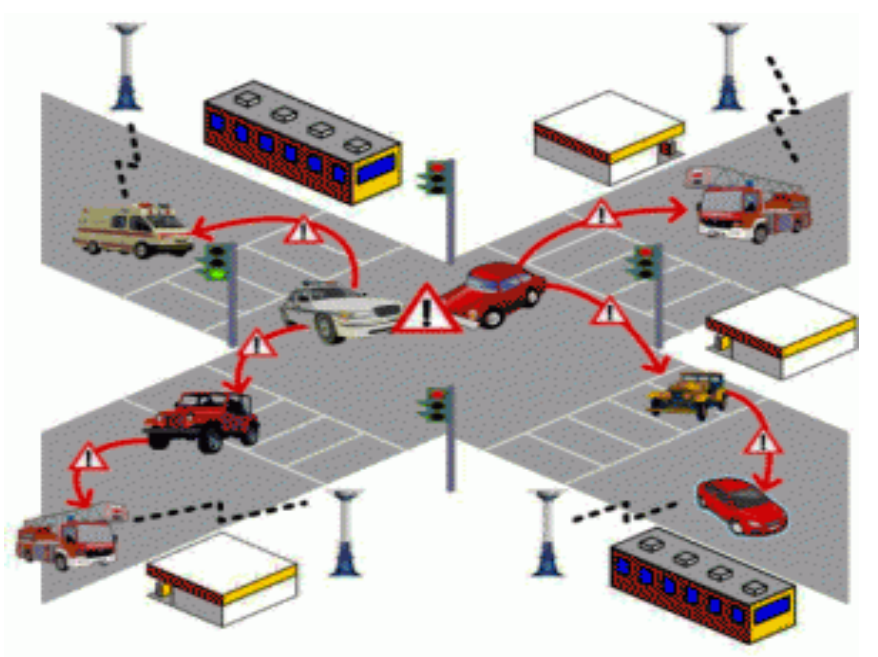

Fig 1: VANET Scenario [6]

\section{VANET SYSTEM ARCHITECTURE}

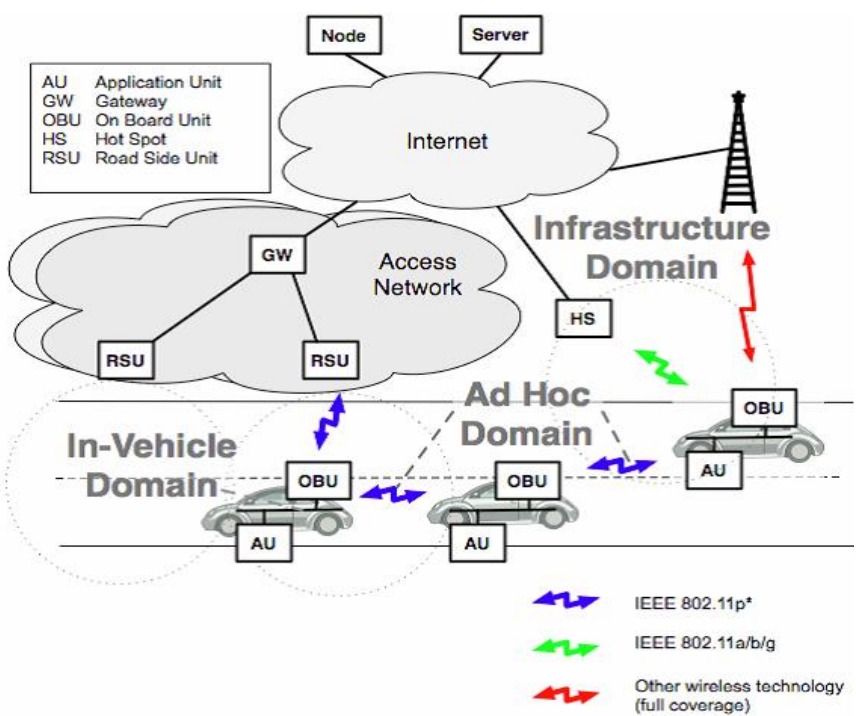

Fig 2: VANET System Architecture

VANET system architecture consists of different domains and many individual components as depicted in Figure2 [7].

\subsection{In-vehicle domain}

This consists of an on-board unit (OBU) and one or more applications units (AU) inside a vehicle. AU executes a set of applications utilizing the communication capability of the OBU. $\mathrm{An} \mathrm{OBU}$ is at least equipped with a (short range) wireless communication device dedicated for road safety, and potentially with other optional communication devices (for safety and nonsafety communications). The distinction between AU and OBU is logical; they can also reside in a single physical unit [7].

\subsection{Ad hoc domain}

An ad hoc domain is composed of vehicles equipped with OBUs and road-side units (RSUs), forming the VANET. OBUs form a mobile ad hoc network which allows communications among nodes without the need for a centralized coordination instance. OBUs directly communicate if wireless connectivity exists among them; else multi-hop communications are used to forward data [7].

\subsection{Infrastructure domain}

The infrastructure consists of RSUs and wireless hotspots (HT) that the vehicles access for safety and non-safety applications. While RSUs for internet access are typically set up by road administrators or other public authorities, public or privately owned hot spots are usually set up in a less controlled environment [7].

\section{APPLICATIONS OF VANET}

The VANET application can be divided into two major categories [10]:

1. Safety and

2. Non-safety.

\subsection{Safety applications}

Safety applications have the ability to reduce traffic accidents and to improve general safety. These can be further categorized as safety-critical and safety-related applications. In the design of security, it should be made sure safety messages are not forged.

\subsubsection{Safety-critical}

These are used in the case of hazardous situations (e.g. like collisions) [11]. It includes the situations where the danger is high or danger is imminent [12]. Safety-critical applications involve communication between vehicles $(\mathrm{V} 2 \mathrm{~V})$ or between vehicles and infrastructure/infrastructure and vehicles (V2I/I2V).

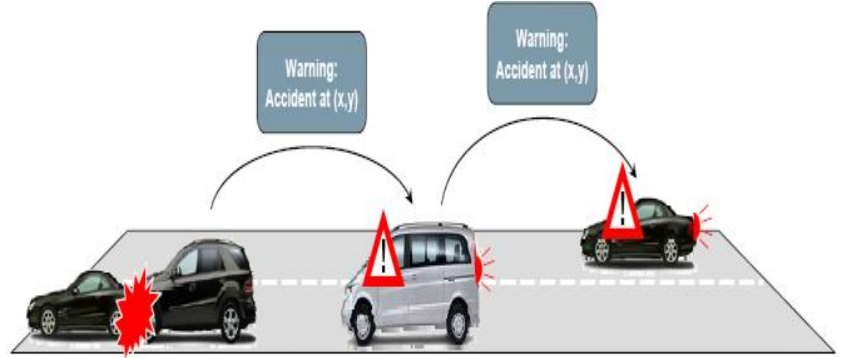

Fig 3: Safety Critical Scenario [16]

\subsubsection{Safety-related}

These include safety applications where the danger is either low (curve speed warning) or elevated (work zone warning), but still foreseeable [12]. In safety-related applications, the latency requirements are not as stringent as in the case of safety-critical ones. Safety-related applications can be V2V or V2I/I2V. 


\section{CSW Curve Speed Warning}

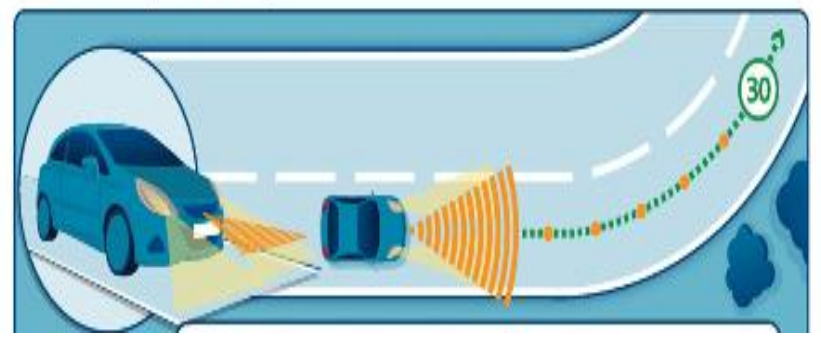

Fig 4: Safety Related Scenario

\subsection{Non-safety applications}

These are applications that provide traffic information and enhance driving comfort. Non-safety applications mostly involve a V2I or I2V communication [10][11]. These services access the channels in the communication system, except the control channel. They access the channel in a low priority mode compared to safety applications.

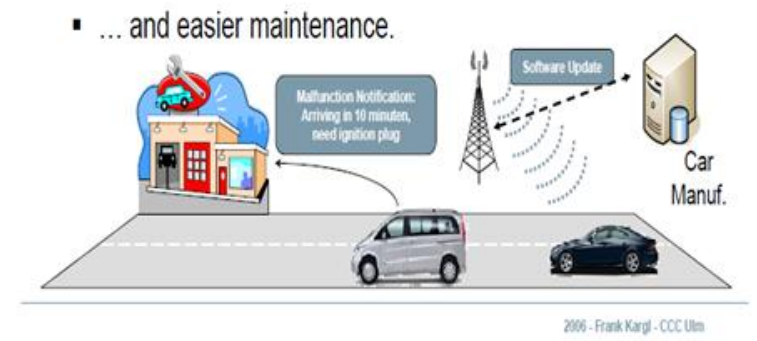

Fig 5: Non-safety Related Scenario [16]

Non-safety applications include applications for[10,11]:

\subsubsection{Traffic optimization}

Traffic information and recommendations, enhanced route guidance etc.

\subsubsection{Infotainment}

The Infotainment services are Internet access, media downloading, instant messaging etc.

\subsubsection{Payment services}

Payment services like Electronic toll collection, parking management etc.

\subsubsection{Roadside service finder}

Finding nearest fuel station, restaurants etc. This involves communication of vehicles with road side infrastructure and the associated database.

\section{ADHOC ROUTING PROTOCOL}

MANETs and VANETs have many similar characteristics, so in this study we are using adhoc routing protocols for the study and analysis of VANET.
Based on the routing strategy the routing protocols can be classified into two parts: 1.Table driven and 2. Source initiated (on demand) while depending on the network structure these are classified as: Flat routing, hierarchical routing and geographic position assisted routing. Flat routing covers both routing protocols based on routing strategy.

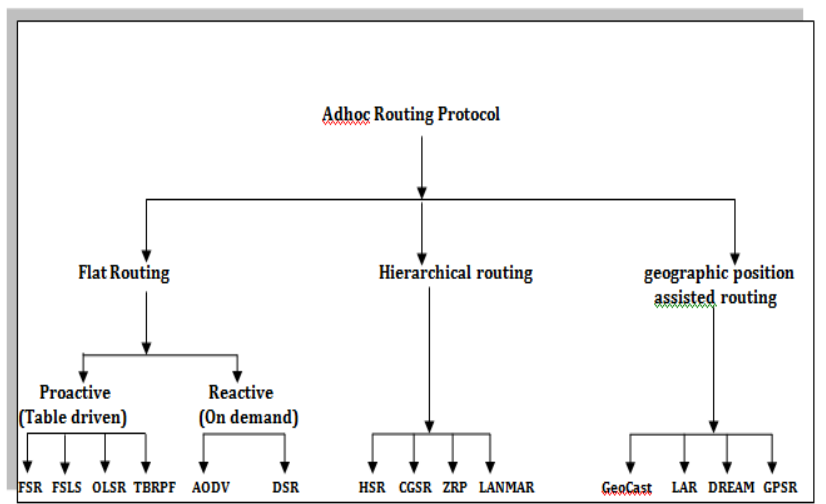

Fig 6: Classification of Adhoc Routing Protocol

In this paper three adhoc routing protocols are used, AODV, OLSR and DSR. AODV and DSR is Reactive (On demand) where as OLSR is Proactive (Table driven) Routing protocol.

\subsection{Ad hoc On-Demand Distance Vector (AODV) Routing Protocol}

The Ad hoc On-Demand Distance Vector (AODV) [8] algorithm enables dynamic, self-starting, multihop routing between participating mobile nodes wishing to establish and maintain an ad hoc network. AODV allows mobile nodes to obtain routes quickly for new destinations, and does not require nodes to maintain routes to destinations that are not in active communication. AODV allows mobile nodes to respond to link breakages and changes in network topology in a timely manner. The operation of AODV is loop-free, and by avoiding the Bellman-Ford "counting to infinity" problem offers quick convergence when the adhoc network topology changes (typically, when a node moves in the network). When links break, AODV causes the affected set of nodes to be notified so that they are able to invalidate the routes using the lost link. Route Requests (RREQs), Route Replies (RREPs) and Route Errors (RERRs) are message types defined by AODV [8].

\subsection{Dynamic Source Routing (DSR)}

The Dynamic Source Routing protocol (DSR) is (Perkins, 2007), an on demand routing protocol. DSR is a simple and efficient routing protocol designed specifically for use in multi-hop wireless ad hoc networks of mobile nodes. Using DSR, the network is completely self-organizing and self-configuring, requiring no existing network infrastructure or administration. The DSR protocol is composed of two main mechanisms that work together to allow the discovery and maintenance of source routes in the ad hoc network [3]:

Route Discovery is the mechanism by which a node $\mathrm{S}$ wishing to send a packet to a destination node $\mathrm{D}$ obtains a source route to $\mathrm{D}$. Route Discovery is used only when $\mathrm{S}$ attempts to send a packet to $\mathrm{D}$ and does not already know a route to D. 
Route Maintenance is the mechanism by which node $\mathrm{S}$ is able to detect, while using a source route to $\mathrm{D}$, if the network topology has changed such that it can no longer use its route to $\mathrm{D}$ because a link along the route no longer works. When Route Maintenance indicates a source route is broken, $\mathrm{S}$ can attempt to use any other route it happens to know to $\mathrm{D}$, or it can invoke Route Discovery again to find a new route for subsequent packets to $D$. Route Maintenance for this route is used only when $\mathrm{S}$ is actually sending packets to D.

In DSR Route Discovery and Route Maintenance each operate entirely" on demand" [ 3].

\subsection{Optimized Link State Routing Protocol (OLSR)}

The Optimized Link State Routing Protocol (OLSR) [9] is developed for mobile ad hoc networks. It operates as a table driven, proactive protocol, i.e., exchanges topology information with other nodes of the network regularly. Each node selects a set of its neighbor nodes as "multipoint relays" (MPR). In OLSR, only nodes, selected as such MPRs are responsible for forwarding control traffic, intended for diffusion into the entire network. MPRs provide an efficient mechanism for flooding control traffic by reducing the number of transmissions required.

Nodes, selected as MPRs, also have a special responsibility when declaring link state information in the network. Indeed, the only requirement for OLSR to provide shortest path routes to all destinations is that MPR nodes declare link-state information for their MPR selectors. Additional available link-state information may be utilized, e.g., for redundancy.

Nodes which have been selected as multipoint relays by some neighbor node(s) announce this information periodically in their control messages. Thereby a node announces to the network, that it has reachability to the nodes which have selected it as an MPR. In route calculation, the MPRs are used to form the route from a given node to any destination in the network.

Furthermore, the protocol uses the MPRs to facilitate efficient flooding of control messages in the network.

A node selects MPRs from among its one hop neighbors with "symmetric", i.e., bi-directional, linkages. Therefore, selecting the route through MPRs automatically avoids the problems associated with data packet transfer over uni-directional links (such as the problem of not getting link-layer acknowledgments for data packets at each hop, for link-layers employing this technique for unicast traffic [9].

\section{RESEARCH METHODOLOGY USED}

The work carried out in this paper is by using open source Simulation tools on realistic scenario of traffic. In this section we are going to describe the working principle of simulation tools used, scenarios used for analysis, simulation setup, metrics used, and performance comparison using excel graphs.

\subsection{Simulation Tools Used}

The simulation tools required for the analysis are mainly classified into two categories and are given as follows:

1. Traffic Simulator

2. Network Simulator
The figure below represents the use of traffic and network simulator used.

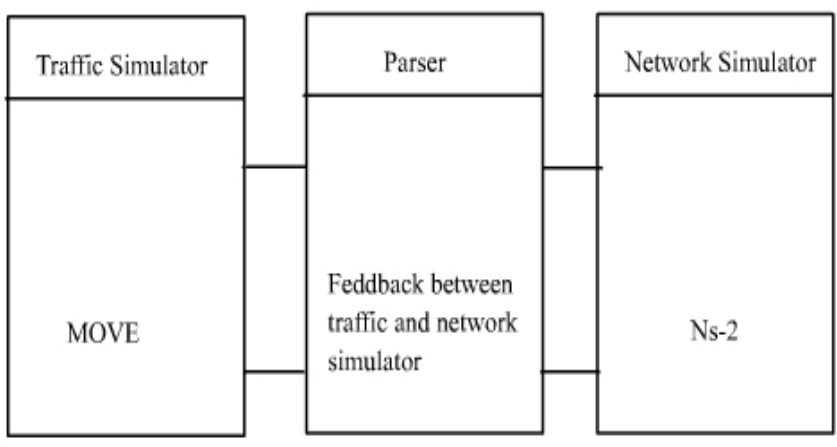

Fig 7: MOVE (Federated)

\subsubsection{MObility model generator for VEhicular networks (MOVE)}

MOVE (MObility model generator for VEhicular networks) is a Java-based application built on SUMO (Simulation of Urban Mobility) with a facility of GUI [13].

MOVE allows users to rapidly generate realistic mobility models for VANET simulations. MOVE is built on top of an open source micro-traffic simulator SUMO.

The output of MOVE is a realistic mobility model and can be immediately used by popular network simulators such as ns-2 and qualnet.

The two main function of MOVE is:

1. MAP Editor

2. Vehicle Movement Editor

The given flow diagram in figure9 represents the main methodology used to perform the simulation using MOVE build on SUMO (Simulation of Urban MObility) and NS2 (Network Simulator 2)[4].

\subsubsection{Simulation of Urban Mobility (SUMO)}

Simulation of Urban MObility" (SUMO) is an open source, highly portable, microscopic road traffic simulation package designed to handle large road networks. It is mainly developed by employees of the Institute of Transportation Systems at the German Aerospace Center [15]. It allows the user to build a customized road topology, in addition to the import of different readymade map formats of many cities and towns of the world.

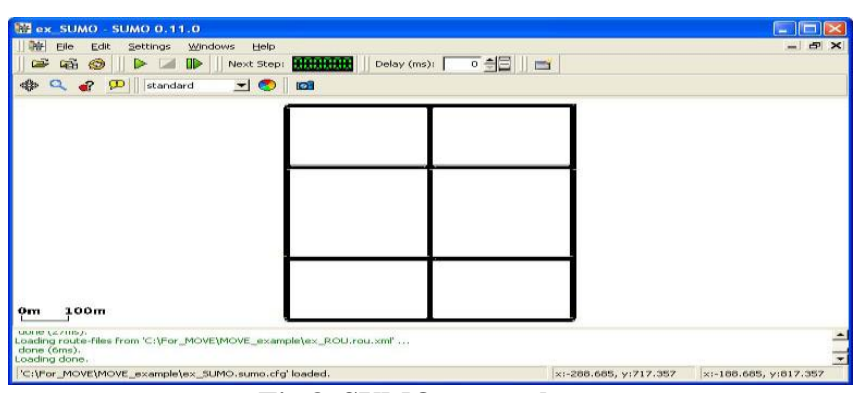

Fig 8. SUMO screenshot 


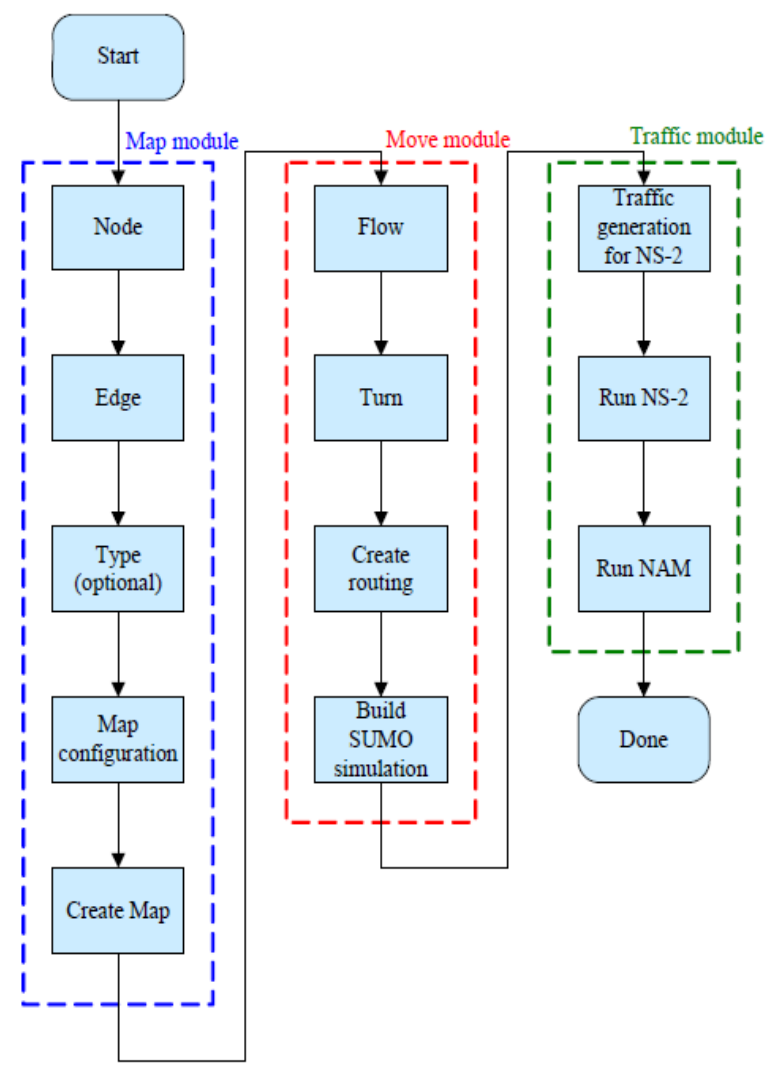

Fig 9: Working of MOVE [14]

\subsubsection{Network Simulator (NS-2)}

NS is a discrete event simulator targeted at networking research. Ns provides substantial support for simulation of TCP, routing, and multicast protocols over wired and wireless (local and satellite) networks [17].

NS2 is an object oriented simulator, written in $\mathrm{C}++$, with an OTcl interpreter as a frontend. This means that most of the simulation scripts are created in Tcl(Tool Command Language). If the components have to be developed for ns 2 , then both tcl and $\mathrm{C}++$ have to be used.

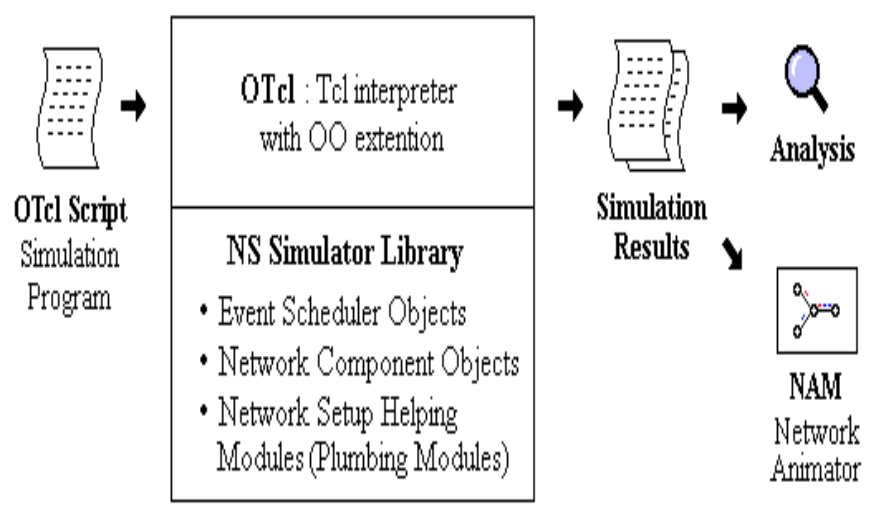

Fig 10: Working of NS-2[18]

\subsection{Simulation Scenario}

The simulation scenario used in this paper is urban and highway scenario both of these are real world scenario downloaded from openstreet map file.

The traffic simulator TraNS(Traffic and Network Simulation Environment)lite[19] over SUMO-0.9.8 is used to generate mobility pattern by converting the real map scenario (exported from open street map). The mobility pattern generated for simulation is totally random. The given scenarios in figure 11 and figure 11 are taken from one of the state of US from urban and highway scenario.

\subsubsection{Urban Scenario}
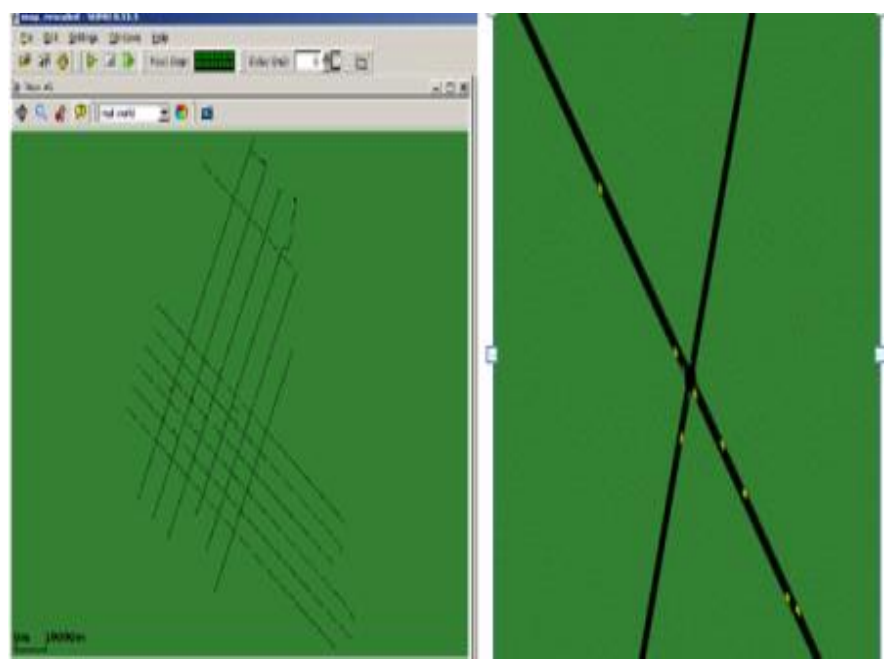

Fig 11: Urban scenario and movement of Vehicles in Urban Scenario (left to right)

\subsubsection{Highway Scenario}

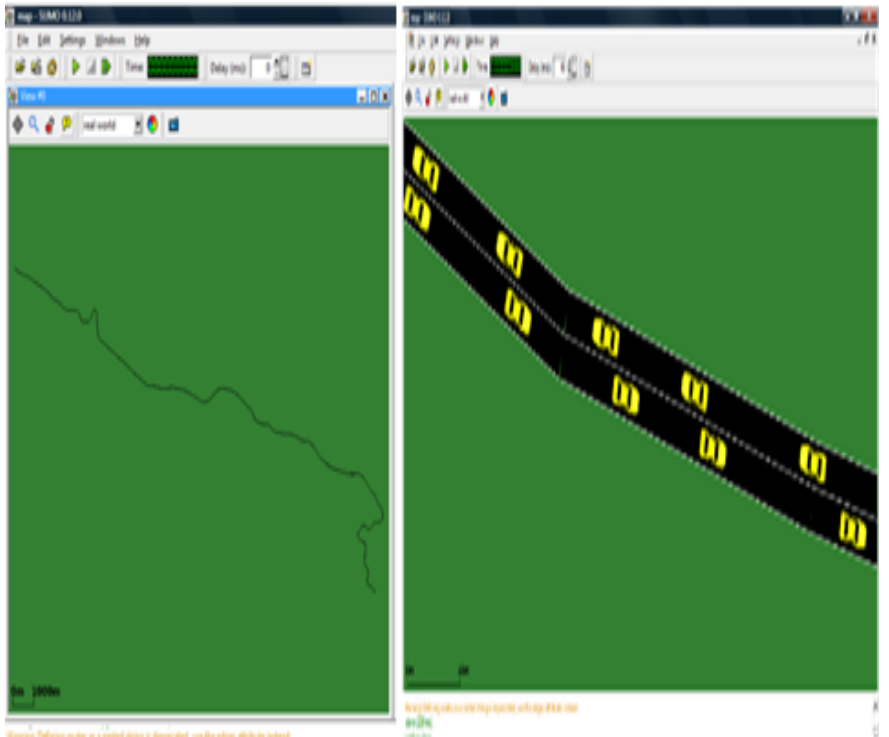

Fig 12: Highway scenario and movement of Vehicles in Highway Scenario (left to right) 


\subsection{Simulation Setup}

The table1 below list the details of simulation setup used in this simulation based analysis.

Table 1. Simulation Setup

\begin{tabular}{|l|l|}
\hline NS version & ns-allinone-2.34 \\
\hline MOVE version & 2.64 \\
\hline SUMO version & 0.9 .8 \\
\hline TraNS version & TraNSlite \\
\hline AODV & NS2 default \\
\hline DSR & NS2 default \\
\hline OLSR & UM OLSR patch \\
\hline Number of Nodes in Urban & $20,102,254,610$ \\
\hline Number of Nodes in Highay & $152,340,767,1216$ \\
\hline Traffic Type & TCP \\
\hline Scenario & Urban \\
\hline Downloaded files & Highway \\
\hline Speed & 40 kmph for Urban and Varying \\
& speed for highway \\
\hline Data type & CBR \\
\hline Data Packet Size & 512 bytes \\
\hline MAC protocol & IEEE 802.11 \\
\hline Radio Propagation Model & Two Ray Ground \\
\hline Simulation Time & 300 seconds \\
\hline $\begin{array}{l}\text { Road Traffic Direction for } \\
\text { Urban }\end{array}$ & Multidirectional \\
\hline $\begin{array}{l}\text { Road Traffic Direction for } \\
\text { Highway }\end{array}$ & Unidirectional \\
\hline No. of Road Lanes & 2 \\
\hline Simulation Area & $\begin{array}{l}5 \mathrm{~km} \text { X 5km for Urban } \\
16 \text { Km for Highway }\end{array}$ \\
\hline & \\
\hline & \\
\hline
\end{tabular}

\subsection{Simulation Metrics used}

The following metrics are used in this paper for the analysis of AODV, OLSR and DSR routing protocols.
i) Packet Delivery Ratio
ii) Average End to End Delay

\subsubsection{Packet Delivery Ratio (PDR)}

The packet delivery ratio in this simulation is defined as the ratio between the number of packets sent by constant bit rate sources (CBR," application layer") and the number of received packets by the CBR sink at destination.

The following equation is used to calculate the PDR,

$\mathbf{P D R}=\left(\sum\right.$ CBR packets received by $\mathrm{CBR}$ sinks

/ $\sum$ CBR packets sent by CBR sources)*100

\subsubsection{Average End to End Delay (Avg. E2E Delay)}

This metric gives the overall delay, from packet transmission by the application agent at the source node till packet reception by the application agent at the destination node. The following equation is used to calculate the average end-to-end delay, Average End-to-End Delay $=\left(T \_D a t a R-T \_D a t a S\right)$, Where
$\mathrm{T} \_$DataR $=$Time data packets received at destination node T_DataS = Time data packets sent from source node.

The end to end delay is important metrics because VANET needs a small latency to deliver quick messages. It shows the suitability of the protocol for the VANET.

\subsection{Simulation Result}

The results are analyzed in different node density and are given as:

$\begin{array}{ll}\text { VLD: } & \text { Very Low Density } \\ \text { LD: } & \text { Low Density } \\ \text { MD: } & \text { Medium Density } \\ \text { HD: } & \text { High Density }\end{array}$

The Density (Concentration) (D) = number of vehicles $(\mathrm{N})$ over a stretch of roadway (L) (in units of vehicles per kilometer) $\mathrm{D}=\mathrm{N} / \mathrm{L}$

\subsubsection{Urban Scenario}

Table 2. Density and Connection pattern used

\begin{tabular}{|c|l|l|l|}
\hline \multirow{4}{*}{$\begin{array}{c}\text { Urban } \\
\text { Scenario }\end{array}$} & Density & No of Nodes & Connections \\
\cline { 2 - 4 } & VLD & 20 & 8 \\
\cline { 2 - 4 } & MD & 102 & 40 \\
\cline { 2 - 4 } & HD & 254 & 100 \\
\hline
\end{tabular}

Figure13 represents the performance of AODV, OLSR and DSR in terms of PDR vs. Node Density.

Figure14 represents the performance of AODV, OLSR and DSR in terms of Average End to End Delay vs. Node Density

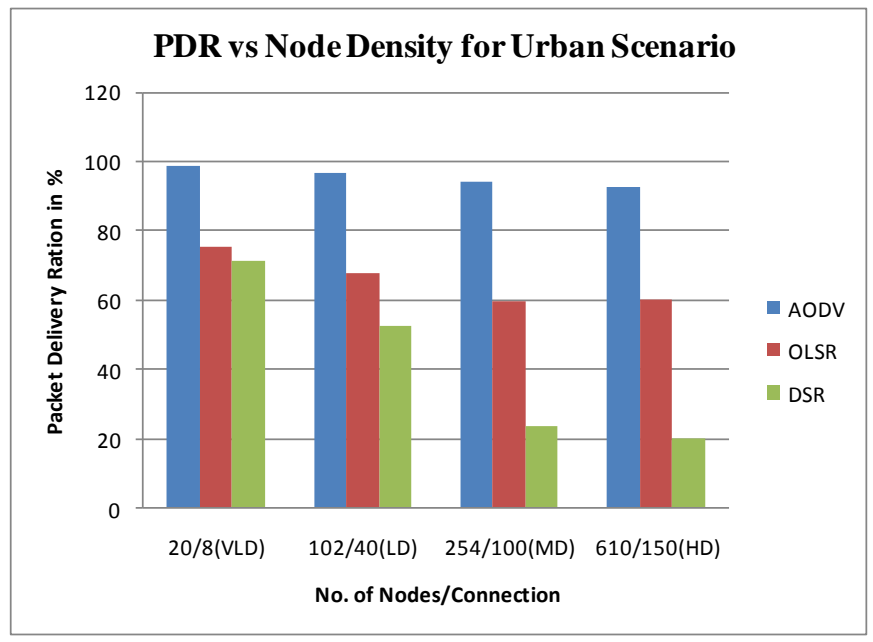

Fig 13: PDR vs. Node Density in Urban Scenario 


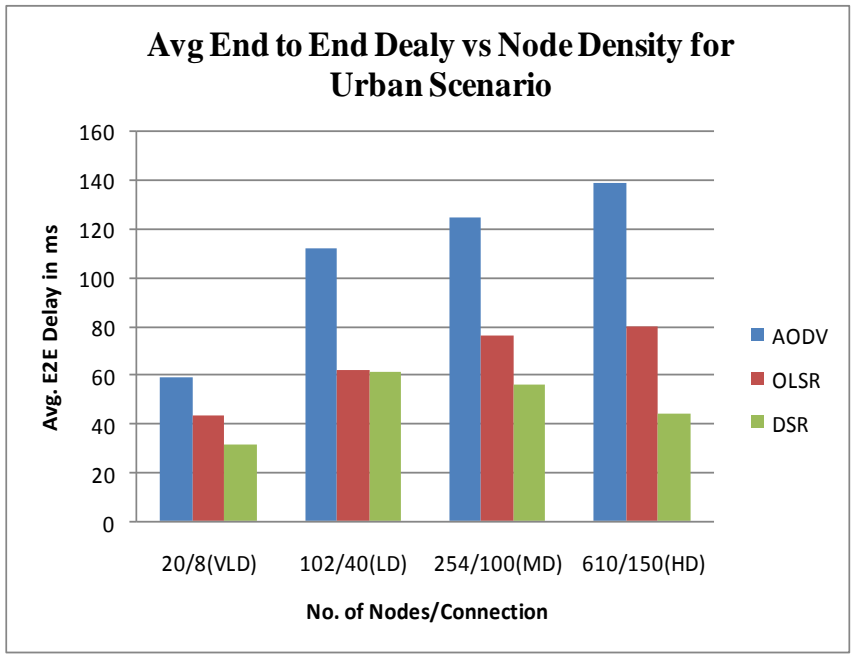

Fig 14: Average End to End Delay (in ms) vs. Node Density in Urban Scenario

\subsubsection{Highway Scenario}

Table3. Density and Connection pattern used

\begin{tabular}{|l|l|l|l|}
\hline \multirow{4}{*}{$\begin{array}{c}\text { Highway } \\
\text { Scenario }\end{array}$} & Density & No of Nodes & Connections \\
\cline { 2 - 4 } & VLD & 150 & 60 \\
\cline { 2 - 4 } & MD & 340 & 120 \\
\cline { 2 - 4 } & HD & 767 & 160 \\
\hline
\end{tabular}

Figure15 represents the performance of AODV, OLSR and DSR in terms of PDR vs. Node Density.

Figure 16 represents the performance of AODV, OLSR and DSR in terms of Average End to End Delay vs. Node Density

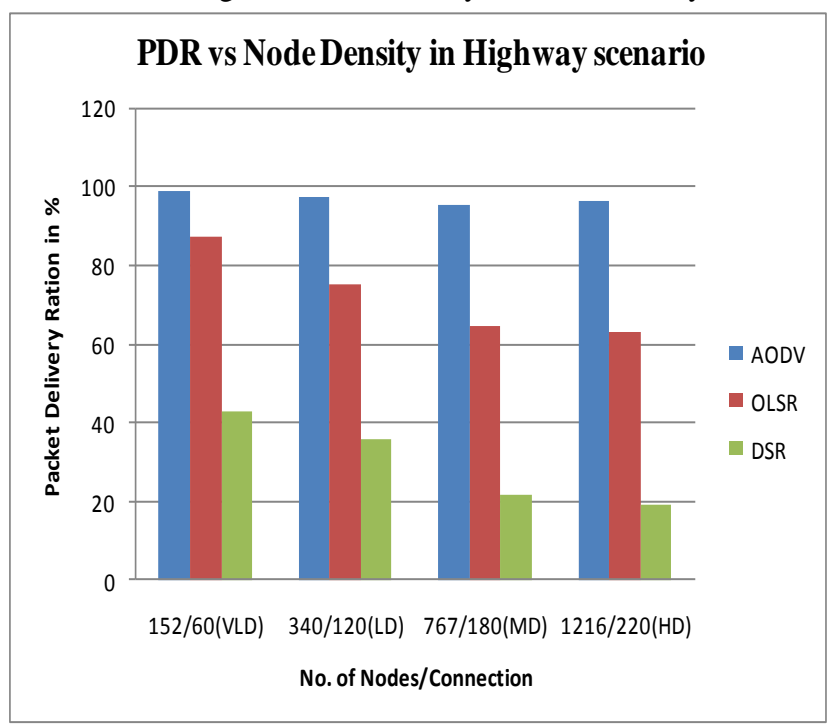

Fig 15: PDR vs. Node Density in Highway Scenario

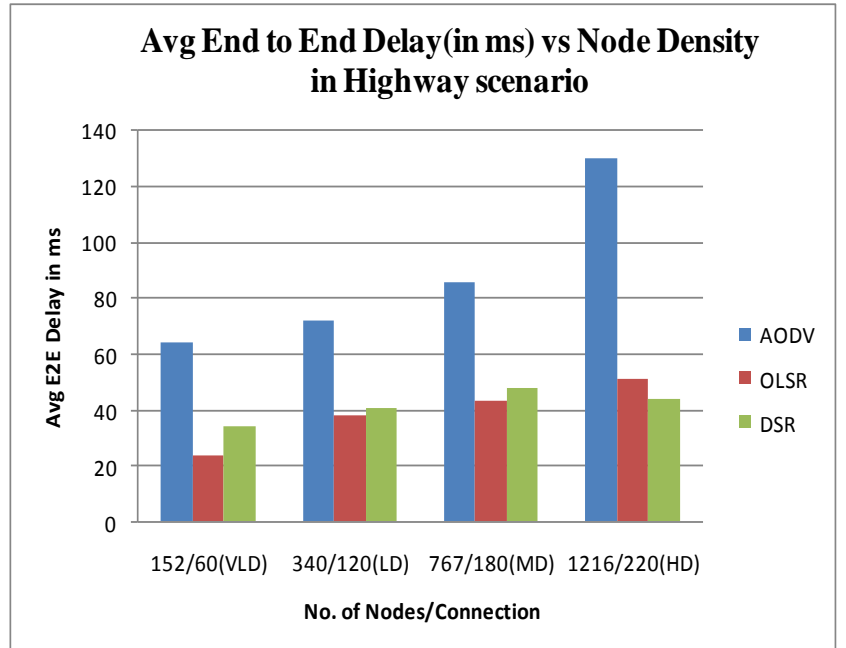

Fig 16: Average End to End Delay (in ms) vs. Node Density in Highway scenario

\section{CONCLUSION}

In this paper the analysis of adhoc routing protocol is done in realistic scenario of VANET. After doing the simulation based analysis of AODV, OLSR and DSR in realistic scenario of VANET we can see that the performance of AODV in terms of PDR is very good approximate $97 \%$ in both scenarios. OLSR has average performance as the PDR is good in Urban but in Highway scenario we found that it decreases as the no. of nodes and connections increases.

The Average end to end delay of AODV is very high in both scenarios for different node densities and reached up to approx $140 \mathrm{~ms}$ in Urban and $130 \mathrm{~ms}$ in Highway scenario. The DSR performs well in both of the scenario in terms of Avg. end to end delay. OLSR is also having low end to end delay in highway but in Urban its performance is not good enough.

Packet delivery Ratio of AODV is better than other two protocols so we can say this protocol is applicable to carry sensitive information in VANET but it fails for the scenario where transmission time should be very less as it has highest end to end delay. For quick transmission DSR performs well but not suitable to carry information as packet loss is very high. The performance of OLSR is average but not suitable to adopt in both scenario of VANET environment.

\section{FUTURE WORK}

In Future we have planned to analyze the performance of routing protocol using NCTUns (National Chiao Tung University Network Simulation) Simulation tool where both traffic and network simulator are tightly coupled together and it supports Intelligent driver model with car following, Intelligent driver model with Lane changing, Intelligent driver model with intersection management. We hope that this will give the real scene of VANET as the vehicles will respond by changing its lane and slowing down speed as in real scenario on road during its movement. We hope the result generated from this tool will be very helpful for the adoption of technology and protocol in VANET. 


\section{REFERENCES}

[1] Hartenstein, H., et al., "Position-Aware Ad Hoc Wireless Networks for Inter-Vehicle Communications: The FleetNet Project," In Proceedings MobiHoc'01: 2nd ACM Int'l. Symp. Mobile Ad Hoc Networking \& Computing, New York: ACM Press, pp. 259-62, 2001.

[2] IEEE Draft P802.11p/D2.0, November 2006. Wireless Access in Vehicular Environments (WAVE).

[3] Mobile Ad Hoc Networking Working Group - DSR, http://www.ietf.org/rfc/rfc4728.txt.

[4] The Network Simulator ns-2. http://www.isi.edu/nsnam/ns/index.html.

[5] Y. Zang, L. Stibor, and H. J. Reumerman, "Neighborhood evaluation of vehicular ad-hoc network using IEEE 802.11p," in Proceedings of the 8th European Wireless Conference, p. 5, Paris, France, 2007.

[6] Vanet Simulator, Report for the Computer Security exam at the Politecnico di Torino Walter Dal Mut, Armand Sofack.

[7] A. Festag, G. Noecker, M. Strassberger, A. Lübke, B. Bochow, M. Torrent-Moreno, S. Schnaufer, R. Eigner, C. Catrinescu, and J. Kunisch. NoW - Network on Wheels: Project Objectives, Technology and Achievements. In Proceedings of 6th International Workshop on Intelligent Transportation (WIT 2008), Hamburg, Germany, Mar 2008. Available at: http://www.elib.dlr.de/48380/1/C2CCC_manifesto_v1.1.pdf

[8] Mobile Ad Hoc Networking Working Group - AODV, http://www.ietf.org/rfc/rfc3561.txt

[9] Mobile Ad Hoc Networking Working Group - OLSR, http://www.ietf.org/rfc/rfc3626.txtl
[10] Vehicle Safety Communications Project Task 3 Final Report. Technical report, The CAMP Vehicle Safety Communications Consortium, Mar 2005. Sponsored by U.S. Department of Transportation (USDOT). Available through National Technical Information Service, Springfield, Virginia 22161.

[11] Rainer Kroh, Antonio Kung, and Frank Kargl. VANETS Security Requirements Final Version. Technical report, Secure Vehicle Communication (Sevecom), Sep 2006. Available at http://www.sevecom.org/Pages/Project Documents.html.

[12] Elmar Schoch, Frank Kargl, Michael Weber, and Tim Leinmuller. Communication Patterns in VANETs. IEEE Communications Magazine, 46:119-125, Nov 2008.

[13] F. Karnadi, Z. Mo, K.-C. Lan, "Rapid Generation of Realistic Mobility Models for VANET", in Proc. of the IEEE Wireless Communication and Networking Conference (WCNC" 07), March 2007.

[14] Rapid Generation of Realistic Simulation for VANET Manual Updated 11 November 2009. Available at: http://www.lens1.csie.ncku.edu.tw/MOVE/Example\%20for\% 20step-by-step.pdf

[15] Vehicular Communications and VANETs Frank Kargl (frank.kargl@ulm.ccc.de) CCC Ulm, Ulm University. Available: http://www.events.ccc.de/congress/2006/Fahrplan lattachments/1216-vanet.pdf

[16] Nsnam web pages: http://www.isi.edu/nsnam/ns/

[17] Sandeep Gupta. "A brief guide to ns2.”

[18] "TRANS - Realistic Simulator for VANETS", [Online]. Available: http://trans.epfl.ch/ 